\title{
Microwave Waveguide-Based Dielectrometry for the Monitoring Erythrocytes' Beta-Receptors Activity
}

\author{
Kateryna Arkhypova, Pavlo Krasov, Anatoliy Fisun \\ Dept. of Solid-State electronics \\ O.Ya. Usikov Institute for Radiophysics and Electronics of \\ the National Academy of Sciences of the Ukraine \\ Kharkov, Ukraine \\ arkhykate@gmail.com
}

\author{
Seil Sautbekov \\ Dept. of Radio engineering, electronics and \\ telecommunications \\ L.N. Gumilyov Eurasian National University \\ Astana, Republic of Kazakhstan
}

\author{
Volodymyr Lychko, Volodymyr Malakhov \\ Dept. of Exercise therapy, sports medicine and rehabilitation \\ Kharkov Medical Academy of Post-Graduate Education \\ Kharkov, Ukraine
}

\begin{abstract}
In the present work we propose a new integrated approach to characterization of cellular reactivity using human erythrocytes in health and disease. We have applied two methods - microwave waveguide-based dielectrometry $(39.5$ GHz) and osmotic fragility technique based on photometry - to monitor receptor-specific response of blood cells caused by the beta-blocker exposure. Our results showed that dielectric response of erythrocytes depends on the physiological state of blood donors that is in good agreement with the degree of their beta-receptors activity (beta-ARM index). We found that druginduced changes in complex permittivity and the values of betaARM index are inversely related. The proposed approach is intended to supplement current cellular analysis used for healthcare, which will allow obtaining more information about health status of the patients at the cellular level.
\end{abstract}

Keywords-permittivity; millimeter wave measurements; photometry; biomedical monitoring; erythrocytes; in vitro; disease

\section{INTRODUCTION}

Scientists currently believe that cellular malfunctions provide the basis of most common diseases $[1,2]$. One of the possible ways to evaluate functional cellular state is to monitor their membrane-receptor activity in the case-control study. In this work, we have focused on the research of erythrocyte membranes activity in patients with ischemic stroke as well as in health individuals. As the target of research is beta-receptor (beta-AR) sensitivity because of its direct involvement in cardio-vascular system and neurological outcome [3-5].

Most of the known methods dealing with cell reception destruct the sample, so the non-invasive microwave techniques may be appropriate. These methods are highly sensitive to structural and functional features of various biological systems. Moreover, they have the essential advantage such as high-speed label-free monitoring in real time.
We propose a new integrated approach to the characterization of erythrocytes' beta-ARs activity. It includes label-free microwave dielectrometry [6] and the modified osmotic fragility [7] methods. Both techniques monitor variation of the parameters being studied in response to the beta-blocker propranolol - a drug widely used in therapy of cardiovascular diseases.

Our investigation has focused on: (1) dielectric behavior of erythrocytes in the case-control study; (2) beta-blockerinduced changes in complex permittivity of erythrocytes of stroke patients within the first 24 hours and on the tenth day of admission; and (3) evaluating beta-ARs of erythrocytes sensitivity to beta-blocker in patients during the course of therapy. We tested the hypothesis whether the values obtained by the two different methods are correlated.

\section{Methods DESCRIPTION AND RESEARCH PROTOCOL}

\section{A. Microwave Waveguide-Based Dielectrometry}

This method is based on the short-circuit waveguide measurements at a fixed frequency of $39.5 \mathrm{GHz}$ ( $\gamma$-dispersion domain). The developed device consists of the reflectometer (see Fig.1) based on the six-port measuring line and a waveguide sensor, which are connected with each other using a clamp. The sensor is a length of $5.2 \times 2.6 \mathrm{~mm}$ waveguide (Fig.2). Such configuration enables to make the repeatable measurements in microsampling mode $(5-6 \mu \mathrm{l})$. A more detailed description of the instrument can be found in [8].

Fig. 3 shows the models of used waveguide sensor. We have found previously that the modification of the reference cuvette (parallel-plate waveguide structure) by additional matching elements leads to the improved measuring performance $[9,10]$. In this case, we used the sensor modified by wedge-shaped dielectric insert (Fig. 3 b). 
Measured complex reflection coefficient $\mathrm{R}^{*}$ is recalculated to the values of complex permittivity $\varepsilon^{*}$ by means of specifically developed software application, which use the experimental data set: calibration results, measurements of the short-circuit load with variable phase, intermediate measurements of loads with known reflection coefficients as well as the test measurements of distilled water and empty cuvette. Further information about the computation algorithm is available in [11].

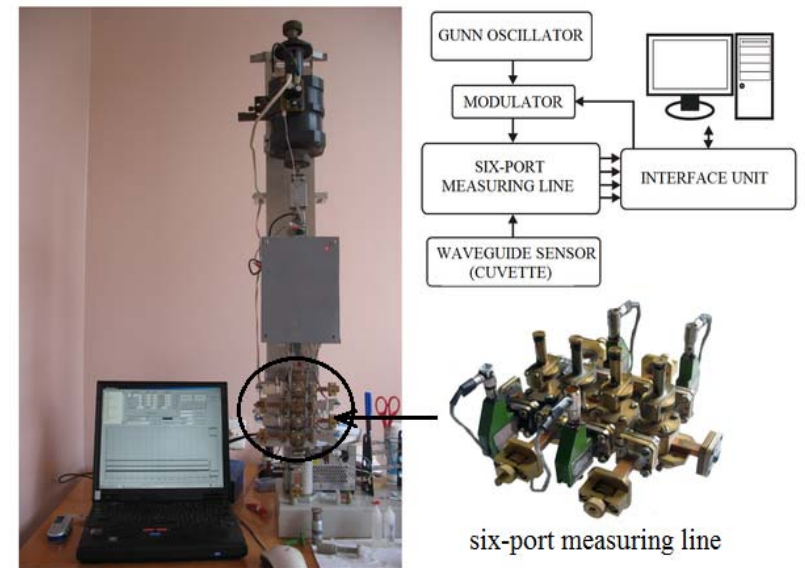

Fig. 1. Measurement setup.

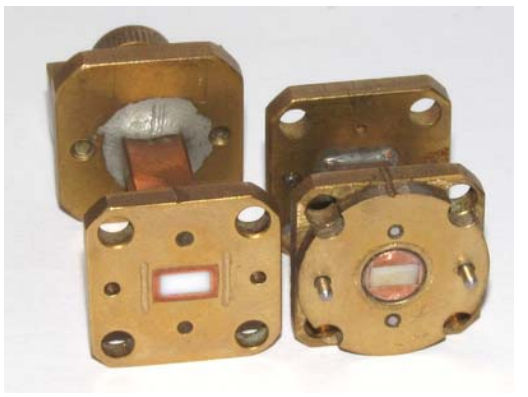

Fig. 2. Exploded view of the waveguide sensor

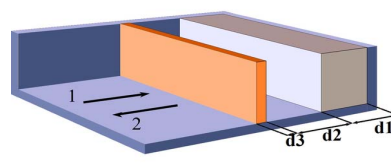

a)

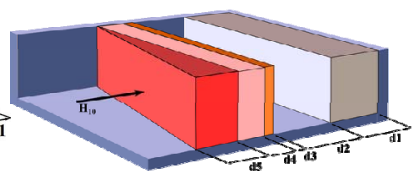

b)
Fig. 3. Sectional view of the waveguide sensor models: reference structure (a) and modified one by the wedge-shaped inserts (b); here $d_{1}-d_{5}$ indicate the thicknesses of dielectric matching inserts. Direction of the incident and reflected waves is shown by arrows 1 and 2, respectively.

\section{B. Osmotic Fragility Method}

It is well-known that erythrocytes placed in a hypotonic medium are exposed to a hemolysis, i.e. to the rupturing of red blood cells and the release of their contents into surrounding fluid. The degree of hemolysis is determined using photocolorimetry method by tracing the optical density of supernatant fluid after blood centrifugation. The basis for the modified method is a hemolysis inhibition in the presence of beta-blocker propranolol, which binds to erythrocytes' beta-ARs and reduces the degree of hemolysis. Receptors activity is numerically expressed by the index of betaadrenergic reactivity of erythrocyte membranes (beta-ARM index), which reflects an increase in osmotic resistance of cell membranes due to the beta-blocker binding. The optical density value of a test sample is expressed as a percentage of the optical density of the control sample and is taken in relative units. About $93 \%$ of healthy individuals have reference values of the beta-ARM index in the range from 2 to 20 relative units [7]. This indicator and the beta-receptors activity have reciprocal correspondence: beta-ARM index will be numerically increased as the osmotic resistance is decreased, and vice versa.

This method is commonly used for the diagnostic of hyper-adrenergic states, prognosis of cardiovascular diseases, and assessment of individual sensitivity to beta-blockers in therapy. We have opted for this technique as a helper for a test case because of its usability and wide application in biomedical research.

\section{Research Protocol}

Measurements were performed using erythrocytes extracted from venous blood of the acute stroke patients $(n=29$, average age is $65.4 \pm 6.3$ years $)$ as well as from health volunteers $(\mathrm{n}=24$, average age is $58.0 \pm 11.3$ years) who were in the control group. Clotting was prevented by the addition of heparin solution $(0.1 \mathrm{ml}$ of 5000 USP units $/ \mathrm{ml})$ for dielectric study, and ethylene diamine-tetracetic acid, or EDTA $(0.1 \mathrm{ml}$ of $0.5 \mathrm{M}$ EDTA per $5 \mathrm{ml}$ of whole blood) for assessing beta-ARM index. More details about the sample preparation can be found in [12]. To characterize erythrocyte beta-ARs activity the cells were exposed to beta-blocker propranolol $(7.5 \mathrm{~g} / \mathrm{l})$ for $30 \mathrm{~min}$. The measurements of permittivity are carried out immediately after the incubation. All manipulations with blood were conducted during 3 hours since its sampling.

Manipulation and statistical data analysis were performed using free software programming language $R$ [13].

\section{RESULTS AND DISCUSSION}

The first step was to observe the dielectric behavior of erythrocytes before and after the exposure to beta-blocker in health and disease. Fig. 4 shows the box-plots representing results of the dielectric measurements of erythrocytes of both group - control and acute stroke patients. The black line in the box indicates the median; the bottom and top of the box indicate the 25 th and 75 th percentiles, respectively. Vertical lines drawn from the box are whiskers. Mean values are asterisked.

Fig. 4 also contains information on the statistical inference (p-values). Between-group differences were analyzed using nonparametric Wilcoxon rank-sum test, whereas the withingroup differences - with use of Wilcoxon sign-rank test. 

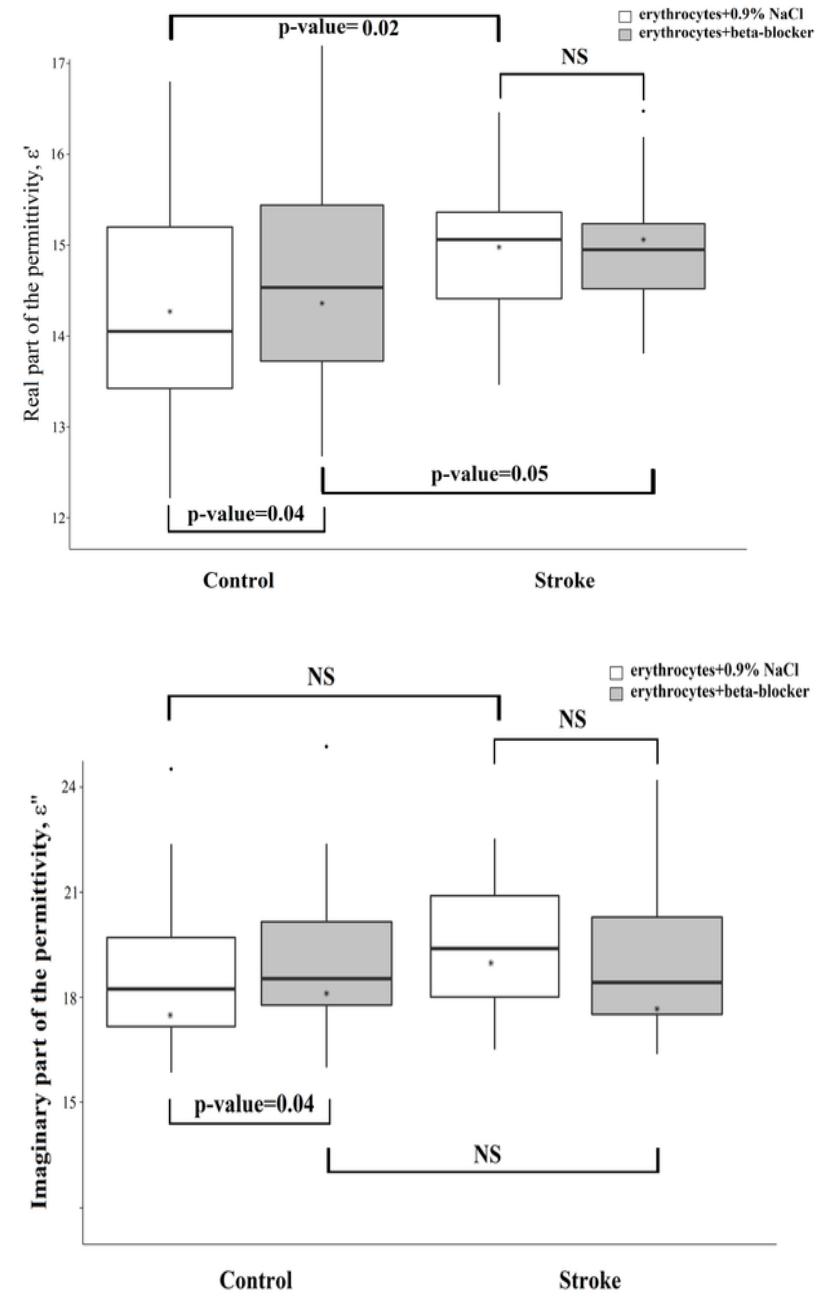

Fig. 4. Complex permittivity of human erythrocytes ( $\mathrm{f}=39.5 \mathrm{GHz}$ at $\left.\mathrm{T}=25^{\circ} \mathrm{C}\right)$ before and after exposure to beta-blocker in control $(\mathrm{n}=23)$ and experimental (stroke, $\mathrm{n}=29$ ) groups.

As we can see, after exposure to beta-blocker, permittivity of erythrocytes in the control group tends to increase (pvalue $=0.04$ ) while in the stroke group it does not change ( $p$ values are not significant - NS). This finding should be interpreted as a lack of cell response to beta-blocker. It can be explained by the fact that drug does not interact with erythrocytes' beta-ARs because of their decreased sensitivity to beta-blocker caused by the disease [7]. These data are in reasonable agreement with the results of the osmotic fragility testing (Fig. 5). It has been shown that a degree of adrenergic activity of erythrocytes in stroke patients significantly reduced compared with ones in the control group; since the values exceeded reference ranges of 2-20 units correspond to a low level beta-receptors activity. Indeed, as Fig.5 shows, we observed highly significant $(\mathrm{p}$-value $<0.001)$ distinction between healthy and diseased groups.

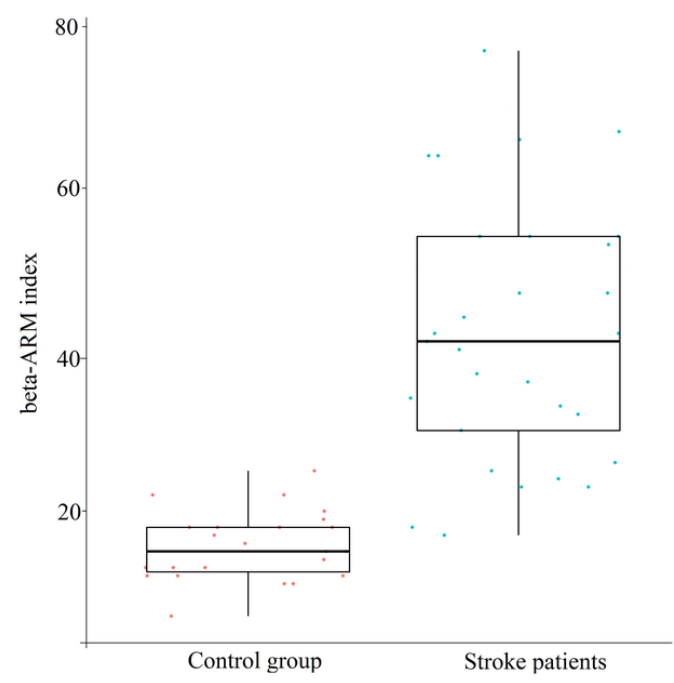

Fig. 5. Experimental distribution of the beta-ARM indices in control $(n=23)$ and stroke patients $(n=29)$ groups.

It should be also noted that significant between-group differences in the absolute values of permittivity (Fig. 4) have been observed in real parts of $\varepsilon^{*}$ only; both differences in imaginary parts are not significant ( $\mathrm{p}$-value $>0.05)$. But, the comparison of the relative values of permittivity (Fig. 6), which represent its difference between values obtained before and after blocker exposure, shows highly significant distinctions $\left(p\right.$-value $=0.01$ and $p$-value $=0.02$ for $\Delta \varepsilon^{\prime}$ and $\Delta \varepsilon^{\prime \prime}$, respectively) between groups. These differences we detected are the cellular response to the drug that depends on the physiological condition of blood donors. And this particular result indicates of high potential of the method for diagnostic purposes.
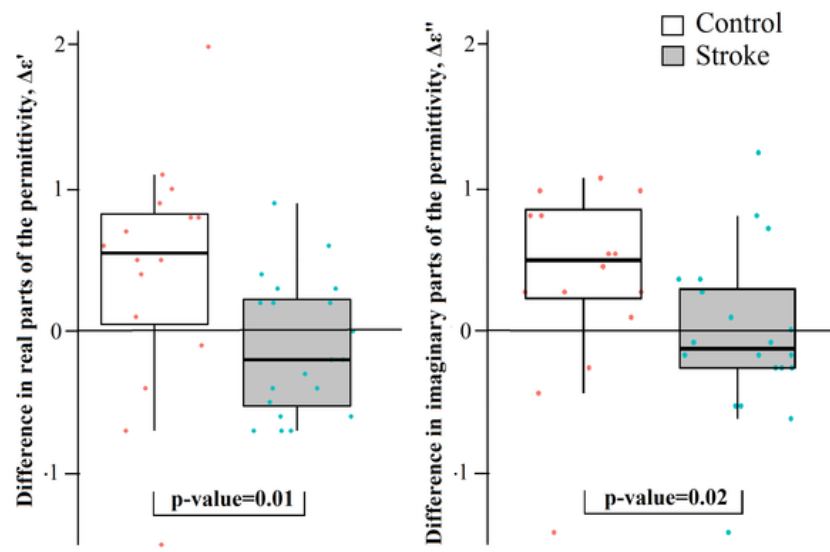

Fig. 6. Differences in the complex permittivity values $\left(\Delta \varepsilon^{\prime}\right.$ and $\left.\Delta \varepsilon^{\prime \prime}\right)$ of erythrocytes caused by the exposure to beta-blocker.

The next step was to characterize the erythrocyte betaARs activity during therapy. Thus, we repeated study on the tenth day of hospitalization. In stroke patients group, we observed a decrease in the beta-ARM index by $30 \%$ (pvalue $<0.03$ ), which indicates normalization of membranes activity and successful therapeutic outcome; while in the 
control group this indicator remained almost unchanged. The permittivity behavior was also improved; the significant differences $(p$-value $<0.05)$ in complex permittivity $\left(\Delta \varepsilon^{\prime}\right.$ and $\left.\Delta \varepsilon^{\prime \prime}\right)$ of erythrocytes after exposure to beta-blocker were increased in $70 \%$ cases, i.e. cell sensitivity to in vitro action of the beta-blockers increased. This result confirms that the values obtained by the two different methods are correlated.

Fig. 7 shows a weak correlation between beta-ARM index and the difference in real parts of permittivity $\Delta \varepsilon^{\prime}$. The correlation coefficient is $r=0.32$ (p-value $=0.03$, Spearman's rank correlation).

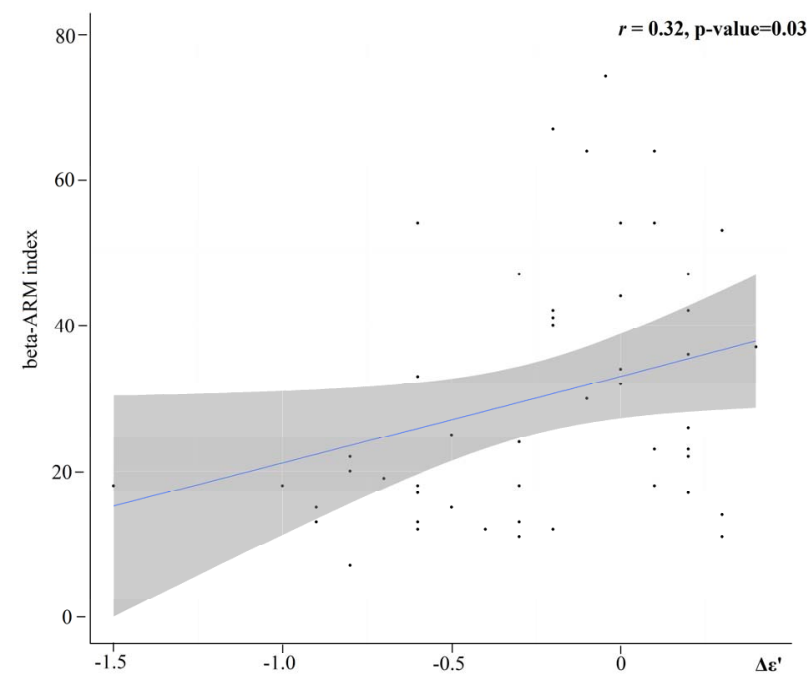

Fig. 7. Spearman's correlation coefficient between beta-ARM index and $\Delta \varepsilon^{\prime}$ of erythrocytes obtained after in vitro beta-blocker exposure

\section{CONCLUSIONS}

In our experiment, we aimed to obtain the evidence of correlation between the dielectric response of blood cells and their beta-receptor-membrane function, which depends on the physiological state of the blood donors. For this purpose, we have integrated two different methods - microwave waveguide-based dielectrometry and the modified osmotic fragility test, and then we performed the measurements using erythrocytes from healthy and diseased groups of individuals.

The results of both methods are shown: (1) clear distinction of the parameters under study between those selected groups; (2) the correlation between the investigated parameters obtained by the two different methods.
We imply that proposed approach may be useful in medical applications for diagnostic purposes. It is intended to supplement current cellular analysis used for healthcare, which will allow obtaining more information about health status of the patients at the cellular level.

\section{REFERENCES}

[1] D.M. Goldberg, J.R. Riordan,"Role of membranes in desease", Clin. Physiol. Biochem, vol. 4(5), pp. 305-36, 1986.

[2] S. Petit-Zeman, "Membranes and the diseases within", Proc. of Horizon Symposia, Nature Publishing Group, pp. 1-3, October 2004.

[3] R.-Q. Han, Yi-B. Ouyang, L. Xu, R. Agrawal, A.J. Patterson, and R.G. Giffard, "Postischemic brain injury is attenuated in mice lacking the 32-adrenergic receptor", Anesth Analg., vol. 108(1), January 2009; pp. 280-287.

[4] Y. Ji, S. Chen, K. Li, X. Xiao, S. Zheng and T. Xu, "The role of $\beta$ adrenergic receptor signaling in the proliferation of hemangiomaderived endothelial cells", Cell Division, vol. 8, pp. 1-11, 2013.

[5] K.V. Luong, L.T.Nguyen, "The role of Beta-adrenergic receptor blockers in Alzheimer's disease: potential genetic and cellular signaling mechanisms", American Journal of Alzheimer's disease and other dementias, August 2013, vol. 28, no. 5, pp. 427-439.

[6] T.Yu. Shchegoleva, Investigation of biological objects in the millimeter-wave range, Kiev; Naukova Dumka, 1996 (in Russian).

[7] R.I. Stryuk, I.G. Dlusskaya, Adrenoreactivity and cardiovascular system, Moskow: Meditsina, 2003 (in Russian).

[8] P.S Krasov and K.A. Arkhipova, "Instrument for measuring the complex permittivity of biological objects", Telecommunications and Radio Engineering, vol. 68, No. 8, pp. 727-733, 2009.

[9] P.S Krasov, K.A. Arkhypova, A.I. Fisun, "Measuring cuvette simulation of dielectrometer for permittivity investigation of high-loss substances", Journal of Measurement Science and Instrumentation, Vol. 2, No. 3, pp. 205-208, 2011.

[10] P.S. Krasov, K.A. Arkhypova, A.I. Fisun, "Cuvette simulation in nonresonator dielectrometer for high-loss substance permittivity measurements", Journal of Applied Electromagnetism, Vol. 13, No. 3, pp. 9-14, 2011 .

[11] K.A. Arkhypova, P.S Krasov, A.I. Fisun, “Application of modified waveguide sensor for dielectric study of red blood cells of patients with discirculatory encephalopathy before and after the therapy", Biophysical Bulletin, Vol. 27, No. 2, pp. 93-102, 2011 (in Russian).

[12] K.A. Arkhypova, "Monitoring the functional properties of human red blood cells by means of waveguide microwave single-frequency dielectrometry method", Telecommunications and Radio Engineering, Vol. 70, No. 6, pp. 547-552, 2011.

[13] I. Fellows, "Deducer: A Data Analysis GUI for R", Journal of Statistical Software, vol. 49(8), pp. 1-15, 2012: http://www.jstatsoft.org/v49/i08/. 